\title{
Protein ubiquitination involving an E1-E2-E3 enzyme ubiquitin thioester cascade
}

\author{
Martin Scheffner*, Ulrike Nuber* \\ \& Jon M. Huibregtse $†$ \\ * Deutsches Krebsforschungszentrum, Angewandte Tumorvirologie, \\ Im Neuenheimer Feld 242, 69120 Heidelberg, Germany \\ † Harvard Medical School, Department of Pathology, \\ 200 Longwood Avenue, Boston, Massachusetts 02115, USA
}

UBIQUITINATION of proteins involves the concerted action of the E1 ubiquitin-activating enzyme, E2 ubiquitin-conjugating enzymes and $\mathrm{E} 3$ ubiquitin-protein ligases ${ }^{1-3}$. It has been proposed that E3s function as 'docking proteins', specifically binding substrate proteins and specific E2s, and that ubiquitin is then transferred directly from $\mathrm{E} 2 \mathrm{~s}$ to substrates ${ }^{1-5}$. We show here that formation of a ubiquitin thioester on E6-AP, an E3 involved in the human papillomavirus E6-induced ubiquitination of p53 (refs 6-10), is an intermediate step in E6-AP-dependent ubiquitination. The order of ubiquitin transfer is from E1 to E2, from E2 to E6-AP, and finally from E6AP to a substrate. This cascade of ubiquitin thioester complexes suggests that E3s have a defined enzymatic activity and do not function simply as docking proteins. The cysteine residue of E6AP responsible for ubiquitin thioester formation was mapped to a region that is highly conserved among several proteins of unknown function, suggesting that these proteins share the ability to form thioesters with ubiquitin.

E6-AP-dependent ubiquitination requires a distinct E2 activity $^{10}$, which is represented by members of a subfamily of E2s that includes Arabidopsis thaliana (At) UBC8 (ref. 11) and human UbcH5 (ref. 12). Coprecipitation analyses, however, revealed no complex between E6-AP and AtUBC8 or UbcH5 (data not shown). This may indicate that the interaction between E6-AP and these E2s is weak and cannot be detected under the conditions of a coprecipitation experiment. Because a necessary step in the ubiquitination of proteins is the E1-dependent attachment of ubiquitin to E2s via a thioester bond ${ }^{1,2,13}$, we tested the effect of E6-AP on the formation of a ubiquitin thioester on AtUBC8 (Fig. 1). In the presence of E1, a product with the characteristics of AtUBC8 linked to ubiquitin by a thioester bond was detected. Complex formation of $\mathrm{E} 2$ with ubiquitin was not significantly affected by the presence of E6-AP, but in the presence of E6-AP an additional complex containing ubiquitin was observed migrating at an approximate relative molecular mass of $130,000(130 \mathrm{~K}$; Fig. $1 a)$. This complex, like the UBC8 ubiquitin complex, had the characteristics of a thioester adduct in that it was labile to reducing agents such as dithiothreitol (DTT) (Fig. 1b) and disappeared on treatment with $0.1 \mathrm{M}$ $\mathrm{NaOH}$ but not with $1 \mathrm{M}$ formic acid (data not shown). The ubiquitin used was a glutathione- $S$-transferase ubiquitin fusion protein (GST-ubiquitin) of $M_{\mathrm{r}} 34 \mathrm{~K}^{10}$. The molecular mass of the recombinant form of E6-AP used in this experiment is $95 \mathrm{~K}^{9}$, suggesting that the $130 \mathrm{~K}$ complex might consist of a single molecule each of E6-AP and GST-ubiquitin. To test this possibility, an amino-terminally truncated form of E6-AP with an $M_{\mathrm{r}}$ of $76 \mathrm{~K}$, which functions in E6-AP-dependent ubiquitination ${ }^{8-10}$, was examined for ubiquitin adduct formation. The $76 \mathrm{~K}$ form of E6-AP and GST-ubiquitin formed a complex of $\sim 110 \mathrm{~K}$ with the characteristics of a thioester (Fig. 1c). Furthermore, as shown previously for E6-AP-dependent protein ubiquitination ${ }^{10}$, E6-AP thioester formation was dependent on the presence of E1 and AtUBC8 or UbcH5 (data not shown). The only other proteins known to form thioesters with ubiquitin are E1 (refs 14, 15) and the members of the E2 family ${ }^{16,17}$, although it was previously noted that E3s might also 
have such an activity ${ }^{18}$. The regions in E1 and the E2s that are responsible for thioester formation have been well characterized $^{2,15}$, but they show little similarity at the protein sequence level. Similarly, protein sequence comparisons did not reveal any significant similarity of E6-AP to either E1 or any of the E2s.

To determine whether thioester formation of E6-AP with ubiquitin correlated with the ability of E6-AP to function as an E3, a deletion mutant of E6-AP lacking the carboxy-terminal 84 amino acids, which can associate with $\mathrm{E} 6$ and $\mathrm{p} 53$ but cannot induce ubiquitination of p53 (ref. 9), was assayed for thioester formation (Fig. 2b). This truncated E6-AP could not form a thioester with ubiquitin (Fig. $2 c$ ), suggesting that the cysteine residue involved in thioester formation may lie in the $\mathrm{C}$ terminus of E6-AP. The $84 \mathrm{C}$-terminal amino acids contain only one cys- teine residue, at position 833 (for numbering, see ref. 8). When this cysteine of the $95 \mathrm{~K}$ form of E6-AP was altered to either serine or alanine, both mutant E6-APs could bind to p53 in the presence of E6 (data not shown), but could not induce ubiquitination of p53 (Fig. $2 b$ ) and did not form a thioester with ubiquitin (Fig. 2c). Thus, the cysteine residue at position 833 of E6-AP is essential for thioester formation as well as for E6-AP-dependent ubiquitination.

Our results indicate that the formation of a ubiquitin thioester on E6-AP is an intermediate step in E6-AP-dependent ubiquitination, and that the order of ubiquitin transfer is from E1 to E2 and then from E2 to E6-AP. This cascade of ubiquitin thioester complexes suggests that ubiquitin is transferred directly from E6-AP to the substrate. In support of this hypothesis, pulse-chase experiments revealed that radioactively labelled
FIG. 1 E6-AP forms a thioester with ubiquitin. $a_{\text {, }}$ Thioester formation of ubiquitin with baculovirusexpressed E6-AP. Thioester reactions contained ${ }^{32}$ P-labelled GST-ubiquitin ${ }^{10}$, E1, Arabidopsis thaliana (At) UBC8, ATP and Mono-Q fractions (see below) containing different amounts of the $95 \mathrm{~K}$ form of E6-AP. Fraction 21 contained the peak of E6-AP activity. As a control, the corresponding fraction of extracts from wild-type baculovirus-infected $\mathrm{Sf9}$ cells was used. After $5 \mathrm{~min}$ at $25^{\circ} \mathrm{C}$, reactions were stopped in the absence of a reducing agent and subjected to SDS-PAGE followed by autoradiography. Because of the low amount of E1 used, the formation of a thioester between ubiquitin and $E 1$ was not detectable. $b$, As $a$, but reactions were stopped in the presence of $100 \mathrm{mM}$ DTT. c, Approximately equal amounts $(150-200 \mathrm{ng})$ of the $95 \mathrm{~K}$ or the $76 \mathrm{~K}$ form of $\mathrm{E} 6-\mathrm{AP}^{9}$ were tested in a thioester assay. Reactions were either stopped in the absence of a reducing agent (-DTT) or in its presence (+DTT). The running positions of GST-ubiquitin (Ub) and the thioester adduct of E2 are indicated. The ubiquitin thioester adducts of the different forms of E6-AP are marked by arrows. Note that formation of a ubiquitin thioester on E6-AP is also observed with native ubiquitin (data not shown).

METHODS. Protein extracts were prepared $48 \mathrm{~h}$ after infection and fractionated by chromatography on Mono- $\mathrm{Q}^{10}$. As $\mathrm{S} f \mathrm{~g}$ cells contain no
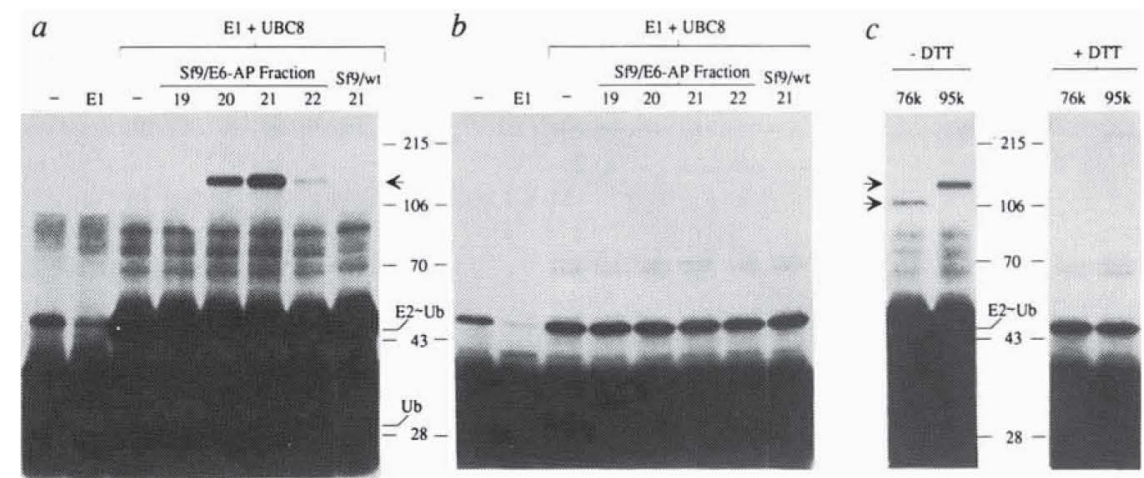

detectable E6-AP activity ${ }^{9}$, fractions containing E6-AP were determined by the ability of these fractions to reconstitute ubiquitination of an E6E7 fusion protein ${ }^{10}$. Thioester reactions contained approximately $1 \mu \mathrm{g}$ ${ }^{32}$ P-labelled GST-ubiquitin, $5 \mathrm{ng}$ of E1 (ref. 15) and $200 \mathrm{ng}$ of AtUBC8 (ref. 11) in $20 \mathrm{mM}$ Tris- $\mathrm{HCl} \mathrm{pH} 7.6,50 \mathrm{mM} \mathrm{NaCl}, 4 \mathrm{mM}$ ATP, $10 \mathrm{mM}$ $\mathrm{MgCl}_{2}, 0.2 \mathrm{mM}$ DTT. Reactions were terminated either by incubating the mixtures for $15 \mathrm{~min}$ at $30^{\circ} \mathrm{C}$ in $50 \mathrm{mM}$ Tris- $\mathrm{HCl} \mathrm{pH} 6.8,2 \%$ SDS, $4 \mathrm{M}$ urea, $10 \%$ glycerol or by boiling the mixtures in the above buffer containing $100 \mathrm{mM}$ DTT instead of urea. The whole reaction mixtures were separated on $10 \%$ SDS-polyacrylamide gels at $4{ }^{\circ} \mathrm{C}$.
FIG. 2 A cysteine residue in the $\mathrm{C}$ terminus of E6-AP is essential for thioester formation. a, Coomassie stain of different mutant forms of E6-AP. Mono-Q fractions containing the $95 \mathrm{~K}$ form of E6-AP, a truncated form of the 95K E6-AP lacking the C-terminal 84 amino acids $(95 \mathrm{k} \Delta \mathrm{C})$, or mutants of the $95 \mathrm{~K} \mathrm{E6-AP}$ in which the cysteine at position 833 was altered to either serine (95k-ser) or alanine (95k-ala) were separated by SDS-PAGE and proteins stained with Coomassie blue. The running positions of $M_{\mathrm{r}}$ markers are indicated. The different forms of E6-AP are marked by arrows. $b$, E6-AP-dependent ubiquitination of p53. Approximately equal amounts of the different forms of E6-AP were tested for their ability to mediate the ubiquitination of p53 in the presence of the HPV-16 E6 protein ${ }^{10}$. c, Thioester formation of the mutated forms of E6-AP. Approximately equal amounts of the different E6-AP were tested in the thioester assay as described for Fig. 1. Reactions were stopped in the absence of a reducing agent. For some E2s, it has been shown that replacement of the active-site cysteine by serine results in the formation of an ester with ubiquitin ${ }^{20}$ which is insensitive to reducing agents. This property, however, is not observed with all members of the E2 family (S. Jentsch, personal communication). The serine $a$

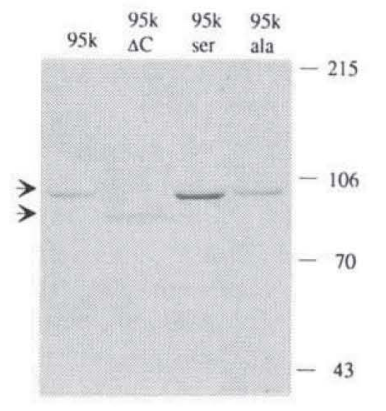

$b$
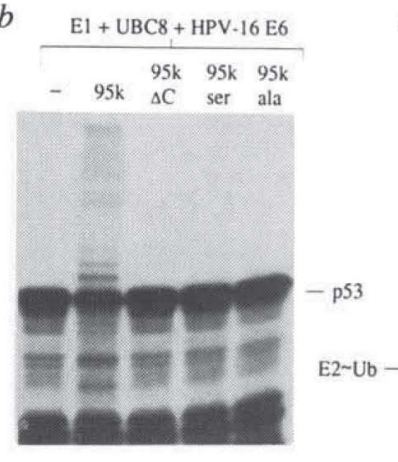

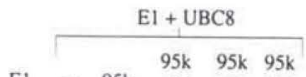

E1

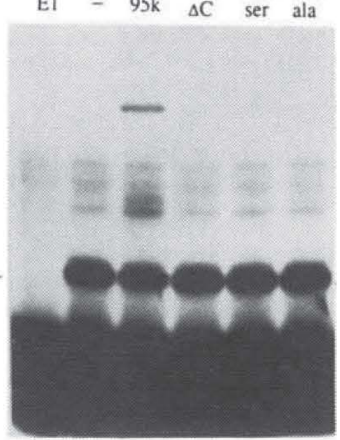

mutant of E6-AP did not form a detectable ester adduct with ubiquitin. METHODS. The construction of the recombinant baculovirus expressing the C-terminally truncated form of the 95K E6-AP has been described ${ }^{9}$. The cysteine residue at position 833 of the E6-AP open reading frame was altered to either serine or alanine by polymerase chain reaction directed mutagenesis. Recombinant baculoviruses expressing these mutated forms of E6-AP were generated as described ${ }^{9}$. 
FIG. 3 Ubiquitin can be directly transferred from E6-AP to substrate proteins. It was previously reported that the Mono-Q fraction containing E6AP also contains proteins that can serve as substrates in E6-AP-dependent ubiquitination ${ }^{10}$. The ubiquitination of these proteins was studied in a pulse-chase experiment. $A$, Reactions were terminated in the absence of a reducing agent. $B$, Reactions were terminated in the presence of $100 \mathrm{mM}$ DTT. E1, AtUBC8, E6-AP and ${ }^{32} \mathrm{P}$ labelled GST-ubiquitin were incubated for $3 \mathrm{~min}$ at $25^{\circ} \mathrm{C}$, resulting in the formation of ubiquitin thioesters on E6-AP (pulse) (lane e). Because of the low amounts of E1 and AtUBC8 used, thioester complexes of AtUBC8 with ubiquitin were barely detectable in the presence of E6-AP. After $3 \mathrm{~min}$ a 50 -fold excess of unlabelled native ubiquitin was added and the reactions were further incubated for different times (chase) (lane e, $0 \mathrm{~min}$; lane $f, 1 \mathrm{~min}$; lane g, $3 \mathrm{~min}$; lane $h, 5 \mathrm{~min}$; lane $i, 10 \mathrm{~min}$ ). Addition of unlabelled native ubiquitin resulted in a significant decrease in the amount of detectable E6AP thioesters, with a concomitant appearance of stably ubiquitinated proteins (marked by a star) migrating at the top of the gel. This strongly suggests that ubiquitin can be transferred directly from E6AP to substrate proteins, although it is not clear whether the final transfer is mediated by E6-AP alone or by a complex consisting of E6-AP and AtUBC8. Running positions of $M_{\mathrm{r}}$ markers, of the thioester of E2 with ubiquitin, and of the thioester of E6-AP with ubiquitin are
B

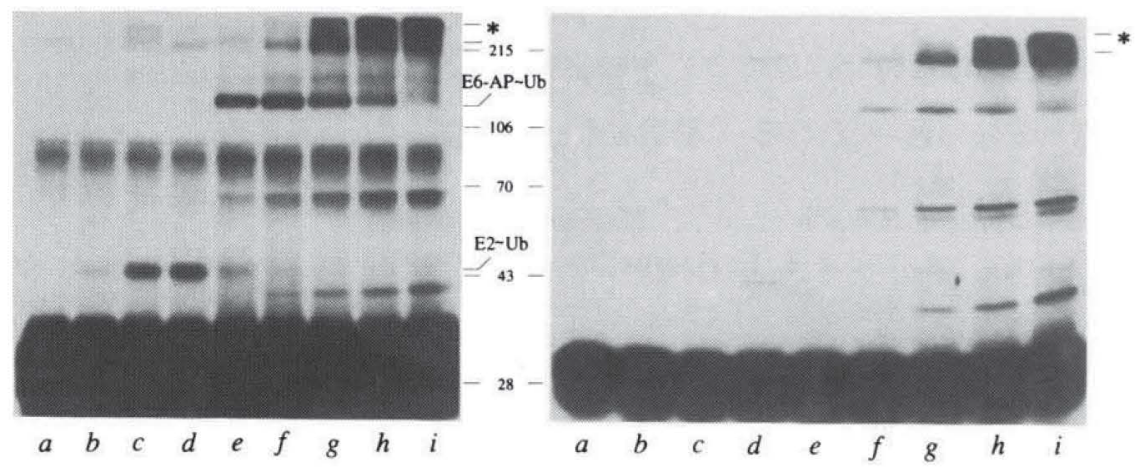

indicated. The identity of thebands migrating at approximately 40 , 66 and $135 \mathrm{~K}$ is unknown (see also ref. 10), but the appearance of these bands is dependent on E6-AP. Lane a, after preincubation of E1 with unlabelled ubiquitin for $3 \mathrm{~min},{ }^{32} \mathrm{P}$-labelled GST-ubiquitin and AtUBC8 were added and the reaction was incubated for an additional 10 min; lane $b$, same as lane a except that E1 was preincubated with ${ }^{32} \mathrm{P}$-labelled GST-ubiquitin and then unlabelled ubiquitin and AtUBC8 were added; lane $c, E 1$ and AtUBC8 were incubated with ${ }^{32} \mathrm{P}$-labelled GST-ubiquitin for $3 \mathrm{~min}$; lane $d$, same as lane $c$ but after 3 min, unlabelled ubiquitin was added and the reaction incubated for an additional $10 \mathrm{~min}$.

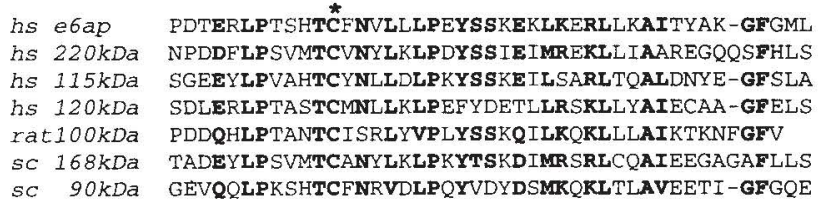

ubiquitin can be chased from E6-AP thioesters to substrate proteins on addition of excess amounts of unlabelled ubiquitin (Fig. 3). Therefore, it seems that E3s do indeed have ubiquitin-protein ligase activity and do not function simply as adaptors between substrate proteins and E2s as previously assumed. Further studies, however, should evaluate whether the proposed mechanism for E6-AP-dependent ubiquitination applies to E3-dependent ubiquitination in general.

Only two E3 genes have been isolated so far, those encoding UBR1 of yeast and E6-AP ${ }^{8,19}$. These proteins have no apparent

\footnotetext{
1. Hershko, A. \& Ciechanover, A. A. Rev, Biochem. 61, 761-807 (1992). 2. Jentsch, S. A. Rev. Genet, 26, 177-205 (1992).

3. Varshavsky, A. Cell 69, 725-735 (1992).

4. Dohmen, R. J., Madura, K., Bartel, B. \& Varshavsky, A. Proc. natn. Acad Sci. U.S.A. 88, $7351-7355$ (1991).

5. Watkins, J. F., Sung, P., Prakash, S. \& Prakash, L. Genes Dev, 7, 250-261 (1993).

6. Scheffner, M., Werness, B. A., Huibregtse, J. M., Levine, A. J. \& Howley, P. M. Cell 63, 1129$1136(1990)$.

7. Huibregtse, J. M., Scheffner, M. \& Howley, P. M. EMBO J. 13, 4129-4135 (1991)

8. Huibregtse, J. M., Scheffner. M. \& Howley, P. M. Molec cell. Biol 13, 775-784 (1993)

9. Huibregtse, J. M., Scheffner, M. \& Howley, P. M. Molec. cell. Biol. 13, $4918-4927$ (1993)

O. Scheffner, M., Huibregtse, J. M., Vierstra, R. D. \& Howley, P. M. Cell 75, 495-505 (1993)

11. Girod, P.-A., Carpenter, T. P., van Nocker, S., Sullivan, M. L. \& Vierstra, R. D. Pl. J. 3, 545552 (1993).

12. Scheffner, M., Huibregtse, J. M. \& Howley, P. M. Proc. natn. Acad. Sci. U.S.A. 91, $8797-$ 8801 (1994).
}

FIG. 4 The region of E6-AP involved in ubiquitin thioester formation is conserved among several proteins from different organisms. A 45-amino-acid overlap that encompasses the $C$ termini of these proteins is shown. Amino acids that are conserved among at least five of the seven proteins are shown in bold type. The cysteine residue of E6-AP involved in ubiquitin thioester formation is marked with an asterisk. The sequences of the rat $100 \mathrm{~K}$ and S. cerevisiae $168 \mathrm{~K}$ have been published ${ }^{21,22}$, while the others were retrieved from the EMBL data library (accession numbers D28476, D25215 and D13635 for Homo sapiens $220 \mathrm{~K}, 115 \mathrm{~K}$ and $120 \mathrm{~K}$, respectively and S53418 for S. cerevisiae 90K).

sequence similarity but databank searches have shown that the $\mathrm{C}$-terminal regions of several proteins from different eukaryotic organisms share significant homology to the $\mathrm{C}$ terminus of E6AP (Fig. 4). The position of the cysteine residue that is essential for thioester formation and for E6-AP-dependent protein ubiquitination, as well as several surrounding residues, are conserved among all these proteins. Although the function of these proteins is unknown, it is tempting to speculate that they share the ability to form thioesters with ubiquitin and are members of an E6-AP like family of ubiquitin-protein ligases.
13. Haas, A. L., Warms, J. V. B., Hershko, A. \& Rose, I. A. J. biol. Chem. 257, 2543-2548 (1982).

14. Ciechanover, A., Elias, S., Heller, H. \& Hershko, A. J. biol. Chem. 257, 2537-2542 (1982) 15. Hatfield, P. M. \& Vierstra, R. D. J. biol. Chem. 267, 14799-14803 (1992) 16. Pickart, C. M. \& Rose, I. A. J. biol. Chem. 260, 1573-1581 (1985).

17. Haas, A. L. \& Bright, P. M. J. biol. Chem. 263, 13258-13267 (1988).

18. Berleth, E. S. et al. J. bjol. Chem. 267, 16403-16411 (1992).

19. Bartel, B., Wünning, 1. \& Varshavsky, A. EMBO J. 9, 3179-3189 (1990)

20. Sung P. Prakash S \& Prakash, L molec Biol 221, 745-749 (1991)

Müller, $D$.

22. Pascolo, S. et al. Yeast 8, 987-995 (1992)

ACKNOWLEDGEMENTS. We thank P. M. Howley, S. Jentsch and K. Münger for critical reading of the manuscript. M.S. thanks S. Stephan for technical assistance and $E$. M. DeVilliers and $H$. zur Hausen for their support. M.S. and J.M.H. thank P. M. Howiey for his support. This work was supported by the Deutsche Forschungsgemeinschaft. 